\title{
Contato pele a pele: conhecimento do profissional de enfermagem
}

\author{
Contact skin skin: knowledge of nursing professional
}

Contacto piel con piel: conocimiento profesional de enfermería

Iara Beatriz Lanaro ${ }^{1}$, Izabel Dayana de Lemos Santos ${ }^{2 *}$, Fabiana Fontana Medeiros ${ }^{2}$, Tatiane Tokushima $^{3}$, Thais da Silva Capello $^{2}$, Mariana Haddad Rodrigues ${ }^{2}$, Rosângela Aparecida Pimenta Ferrari $^{2}$, Alexandrina Aparecida Maciel Cardelli².

\section{RESUMO}

Objetivo: Compreender o conhecimento do trabalhador da área de enfermagem em relação ao contato pele a pele. Métodos: Trata-se de uma pesquisa com abordagem qualitativa. Para compreensão dos relatos, foi utilizada a análise de conteúdo de Bardin sendo dividida em: pré-análise, leitura flutuante, exploração do material e tratamento dos resultados. Resultados: A análise das entrevistas permitiu o delineamento de três categorias temáticas, sendo elas: A fisiologia como base para o contato pele a pele; Conforto e vínculo representados pelo contato pele a pele; O conhecimento e a prática da técnica do contato pele a pele em recém-nascido de alto risco. Conclusão: A partir da compreensão do conhecimento do profissional de enfermagem percebeu-se a necessidade de uma reflexão aprofundada, por parte do profissional da área de enfermagem, sobre o contato pele a pele, a sua prática e seus benefícios, mesmo assim, a técnica é efetuada corretamente quando avaliada a sua possibilidade de acontecer.

Palavras-chave: Gravidez de alto risco, Enfermagem obstétrica, Enfermagem materno-infantil.

\section{ABSTRACT}

Objective: To understand the knowledge of the nursing worker in relation to skin-to-skin contact. Methods: This is a research with a qualitative approach. To understand the reports, Bardin's content analysis was used and divided into: pre-analysis, floating reading, material exploration and treatment of results. Results: The analysis of the interviews allowed the delineation of three thematic categories, namely: Physiology as a basis for skin-to-skin contact; Comfort and bonding represented by skin-to-skin contact; Knowledge and practice of skin-to-skin contact in high-risk newborns. Conclusion: From the understanding of the knowledge of the nursing professional, it was realized the need for an in-depth reflection, on the part of the nursing professional, about skin-to-skin contact, its practice and its benefits, even so, the technique it is carried out correctly when evaluated its possibility of happening.

Keywords: High risk pregnancy, Obstetric nursing, Maternal-child nursing.

\section{RESUMEN}

Objetivo: Comprender los conocimientos del trabajador de enfermería en relación al contacto piel con piel. Métodos: Se trata de una investigación con enfoque cualitativo. Para la comprensión de los informes se utilizó el análisis de contenido de Bardin y se dividió en: preanálisis, lectura flotante, exploración material y tratamiento de resultados. Resultados: El análisis de las entrevistas permitió delinear tres categorías temáticas, a saber: Fisiología como base para el contacto piel a piel; Comodidad y unión representada por el contacto piel a piel; Conocimiento y práctica del contacto piel a piel en recién nacidos de alto riesgo. Conclusión: A partir de la comprensión de los conocimientos del profesional de enfermería, se comprendió la necesidad de una reflexión profunda, por parte del profesional de enfermería, sobre el contacto piel a piel, su práctica y sus beneficios, aun así, la técnica. se lleva a cabo correctamente cuando se evalúa su posibilidad de ocurrir.

Palabras clave: Embarazo de alto riesgo, Enfermería obstétrica, Enfermería maternoinfantil.

${ }^{1}$ Hospital Pequeno Príncipe, Curitiba - PR.

2 Universidade Estadual de Londrina (UEL), Londrina - PR. *E-mail: izabellemos87@hotmail.com

${ }^{3}$ São Francisco Instituto Vida, Cambé - PR.

SUBMETIDO EM: 9/2020

ACEITO EM: 10/2020

PUBLICADO EM: 1/2021 


\section{INTRODUÇÃO}

O modelo hospitalocêntrico de parir do início do século XX até a década de 30 instituiu ambientes estéreis e antissociais para a recepção do recém-nascido (RN). Salas anexas às salas de parto foram criadas com o objetivo de que os procedimentos realizados no RN tivessem o índice de infecções reduzido, separando binômio (mãe e bebê) no instante do nascimento. Os procedimentos de rotina eram realizados em berçários que mantinham os bebês afastados de suas mães. Após o retorno materno para enfermaria os RNs eram nutridos com leite materno e em seguida retornavam aos cuidados do berçário (ROSA HR, et al.,2016).

Em 1990, o Brasil foi um dos doze países participantes do encontro promovido pela Organização Mundial da Saúde (OMS) e o Fundo das Nações Unidas para a Infância (UNICEF) em Florença, Itália. No encontro, um conjunto de metas chamado "Declaração de Innocenti" foi criado com o intuito de reverter os altos índices de desmame precoce e contribuir para a diminuição da mortalidade infantil.

Foi apresentada, também, neste mesmo encontro a "Iniciativa Hospital Amigo da Criança" que visa promover, proteger e apoiar o aleitamento materno e ao "Aleitamento Materno na Década de 90: uma Iniciativa Global" que defende o direito da mulher de realizar a amamentação com sucesso. A recomendação sugerida no encontro era que a mulher mantivesse o aleitamento materno exclusivo até os seis meses de vida do bebê, e, se possível, que ela mantivesse o aleitamento materno em conjunto a outros alimentos até os dois anos de idade da criança (LAMOUNIER JA, 2019; WORLD HEALTH ORGANIZATION, 2017).

Mesmo que no encontro de Florença houvesse apenas 12 países reunidos, a Iniciativa Hospital Amigo da Criança é, atualmente, uma estratégia mundialmente aplicada. Um conjunto de medidas denominado "Dez Passos para o Sucesso do Aleitamento Materno" foi estruturado formalmente para que ao menos, os países do encontro às tornassem realidade em seus hospitais (LAMOUNIER JA, 1996).

Considerando a abordagem da "Iniciativa Hospital Amigo da Criança" , que tem uma importância significativa na atenção à saúde infantil de alcance mundial, ressalta-se a prática do contato pele a pele como um facilitador para a amamentação na primeira hora pós-parto, lembrando que quando a amamentação é estabelecida logo na primeira hora pós parto, as chances do aleitamento materno exclusivo (AME) se estabelecer é maior e assim a criança recebe inúmeros benefícios que se iniciam logo após o nascimento e se estendem para vida toda (SILVA CM, 2016).

O quarto passo conforme a nova interpretação, visa o contato pele a pele imediato após o parto por ao menos uma hora consecutiva, sendo benéfico tanto para a mãe quanto para o RN. Um dos maiores benefícios para o binômio mãe e bebê, em relação ao contato pele a pele é o comportamento pré-alimentar organizado que o RN cria enquanto localiza a mama, iniciando movimentos de busca espontâneos e sucção, abocanha o mamilo e suga, desta forma, a amamentação na primeira hora e contato pele a pele complementam-se (WORLD HEALTH ORGANIZATION, 2018).

Diante do cenário exposto, observou-se a necessidade de um novo modelo de parir, desta forma, a promoção do contato pele-a-pele imediato após o parto tem sido objeto de estudos e trabalhos científicos, uma vez que realizado, favorece o início da amamentação, além de outros benefícios associados para mãe e bebê, tais como: aquecimento do RN, regulação cardiorrespiratória do $\mathrm{RN}$, assim como vinculo da família constituída naquele momento (MATOS TA, et al., 2010; COCA KP, et al., 2018).

O contato pele a pele é definido como o contato do binômio, mãe e bebê, imediatamente após o parto por uma hora contínua e prolongada, em temperatura ambiente de $26^{\circ} \mathrm{C}$, o bebê de bruços pele com pele sob 0 tórax ou abdome materno com as costas do $\mathrm{RN}$ coberto por campos preaquecidos, sem intervenções médicas invasivas, porém com supervisão, para detecção precoce de qualquer tipo de anormalidade. Desta forma a primeira amamentação também é estimulada (BRASIL, 2012).

O RN necessita de avaliação de sua vitalidade no momento do nascimento, sendo avaliados os seguintes pontos: se a gestação é a termo; se o bebê está respirando ou chorando; se o bebê tem tônus muscular adequado. Caso estejam respondidas positivamente e os parâmetros dos sinais estejam de acordo com os preconizados pelo ministério da saúde, considera-se que o RN não necessita de manobras de reanimação, dessa maneira, um RN com boa vitalidade está apto a ser colocado em contato pele a pele com mãe (BRASIL, 2012; SOCIEDADE BRASILEIRA DE PEDIATRIA, 2016). 
O contato pele a pele traz benefícios tanto para o $\mathrm{RN}$ como para puérpera, benefícios reais, imediatos e futuros, desde fisiológicos como a melhor adaptação extrauterina para o neonato referente a regulação termodinâmica, cardiorrespiratória, imunológica e nutricional, assim como para puérpera na descarga hormonal da ocitocina, que tem ação direta na liberação do leite materno e na contratilidade uterina, deste modo prevenindo hemorragias pós parto por atonia uterina e favorecendo melhor involução do útero , até as de cunho afetivo, é neste momento que a mãe tem o primeiro contato com seu bebê e o vinculo é estabelecido de maneira positiva (BRASIL, 2011)

Mediante seus benefícios, o contato pele a pele tem ganhado espaço nas maternidades e sua importância vem sendo reconhecida por evidências cientificas, mas ainda existem lacunas no método de aplicação, eficácia e reais benefícios do mesmo, podendo gerar resistência dos profissionais para sua prática. Deste modo, este estudo teve como objetivo compreender o conhecimento do trabalhador da área enfermagem em relação ao contato pele a pele no pós-parto imediato de acordo com as políticas públicas vigentes.

\section{MÉTODOS}

Trata-se de uma pesquisa com abordagem qualitativa, realizada em um hospital terciário credenciado ao Sistema Único de Saúde (SUS), vinculado a uma universidade estadual, que realiza assistência a partos de alta complexidade e atendimento os recém-nascidos de risco. A instituição possui certificado amigo da criança, uma estratégia da OMS e UNICEF, com objetivo de intensificar o estímulo ao aleitamento materno exclusivo até os seis meses de vida, com a introdução de alimentação complementar de forma e tempo adequado (BRASIL, 2014).

O estudo foi realizado na maternidade da instituição. A população do estudo foi composta pelos trabalhadores da área de enfermagem do setor. Os critérios de inclusão adotados na seleção da amostra foram: funcionários que trabalhem há um ano na unidade, que estejam inseridos na escala fixa do setor e que tenham contato direto com mãe e RN no momento do parto normal. Os critérios de exclusão foram: funcionários de licença prêmio, licença por motivos de saúde e funcionários de férias. $O$ quadro de funcionários da instituição conta com 20 integrantes, sendo estes exclusivamente mulheres, entre elas enfermeiras e técnicas de enfermagem.

Após levantar o número de funcionárias, foram feitas visitas em todos os turnos de trabalho da unidade pela pesquisadora principal, tendo em vista o vínculo inicial com as potenciais participantes do estudo, em seguida foi explicado o objetivo da pesquisa para cada uma, pessoalmente e individualmente, em seguida convidadas a participarem do estudo por intermédio de entrevistas gravadas. No entanto nenhuma das funcionárias aceitaram participar da pesquisa, alegando não ter tempo para colaborar com o estudo, pois dentre as profissionais convidadas uma parte desenvolvia suas funções em duas instituições, as demais faziam horas extras na própria instituição, além daquelas que não tinham interesse em contribuir com a pesquisa.

Frente a este parecer, foram ofertadas várias outras maneiras de se obter as entrevistas tais como: visita domiciliar aos participantes e a solicitação para chefia que as participantes pudessem responder a entrevista no período de trabalho, nenhuma das propostas foram aceitas pela população elegível selecionada, porém ainda existia a inquietação sobre o tema exposto, foram necessárias adaptações na coleta dos dados, então um formulário semiestruturado foi elaborado contendo as questões norteadoras, no qual as participantes teriam liberdade de escrever suas respostas e fariam no momento mais oportuno para cada uma, desta forma cinco colaboradoras aceitaram participar da pesquisa.

A coleta de dados foi realizada no período de junho a agosto de 2017. Os formulários foram entregues às funcionárias em mãos no próprio ambiente de trabalho, com as seguintes questões norteadoras: Qual sua experiência pessoal com o contato pele a pele? O que é contato pele a pele? Qual o seu conhecimento sobre o contato pele e pele nos partos de alto risco? Qual sua visão relativa à necessidade de haver o contato pele a pele em partos de alto risco? Qual frequência em que você colabora para que aconteça o contato pele a pele no parto de alto risco? Para compreensão dos relatos foi utilizada a análise de conteúdo que visa produzir inferências do conteúdo da comunicação de um texto, replicáveis ao seu contexto profissional. 
Para Bardin, a análise de conteúdo basicamente desdobra-se em três fases: a pré-análise que compreende o momento de organização do material, leitura flutuante e exploração da hipótese baseada nos relatos; a exploração do material, momento em que se realiza o agrupamento dos discursos, com intuito de responder o objetivo da pesquisa em formas de categorias; e o terceiro é o tratamento dos resultados, estágio que acontece a inferência e a interpretação do conteúdo (BARDIN L, 2011).

As cinco funcionárias que concordaram participar da pesquisa assinaram o Termo de Consentimento Livre e Esclarecido em duas vias, permanecendo uma via com a participante e a outra com o pesquisador. Foi garantido o sigilo sobre sua identidade no estudo, a letra $E$ de enfermagem foi utilizada para assegurar e preservar a identidade do participante Ex: E1. Este estudo foi submetido a do Comitê de Ética em Pesquisa da Universidade Estadual de Londrina, que trata de pesquisa envolvendo seres humanos. Com número do parecer 1.757.596 e CAAE 59935716.7.0000.5231.

\title{
RESULTADO E DISCUSSÃO
}

A análise de dados permitiu o delineamento de três categorias temáticas, emergentes das respostas dos instrumentos fornecidos as profissionais de enfermagem que aceitam participar da pesquisa, sendo elas: $\mathrm{A}$ fisiologia como base para o contato pele a pele; Conforto e vínculo representados pelo contato pele a pele; $O$ conhecimento e a prática da técnica do contato pele a pele em $\mathrm{RN}$ de alto risco.

\section{A fisiologia como base para o contato pele a pele}

Nas respostas dos membros da equipe de enfermagem constatou-se que o embasamento teórico da fisiologia do contato pele a pele na prática existe, porém fragmentada e pouco explorada, demonstrada em uma dificuldade de aprofundamento cientifico nas falas. Observado a seguir:

\author{
"Eu vejo sempre uma grande melhora na hipotermia." (E3) \\ "O RN se recupera mais rápido em contato com a mãe." (E5) \\ "[...] contato sem roupa do RN com a pele da mãe, com uma malha de algodão, com \\ a finalidade de aquecer." (E3)
}

O apontamento do benefício na melhora da hipotermia citado nas respostas, corrobora com o que está descrito na literatura, sendo um benefício imediato para o recém-nascido à regulação da temperatura corporal, componente essencial para a prevenção da morbidade neonatal, salientando que o feto está adaptado a temperatura corporal de sua mãe enquanto vida intrauterina uma exposição extrema a baixas temperaturas, tem como resposta no metabolismo do recém-nascido um grande gasto energético para manutenção da temperatura e em decorrência de um consumo energético excessivo neste momento o recém-nascido pode apresentar hipoglicemia, tornando sua adaptação extrauterina mais difícil, porém, nota-se falta de conhecimento dos demais benefícios ao lactente e sua genitora em níveis fisiológicos (BRASIL, 2013; SOARES T, 2020).

Esperava-se encontrar como maior benefício mencionado pelas entrevistadas a amamentação na primeira hora de vida, por este tema ser predominante na literatura, porém em momento algum foi citada a amamentação como aspecto benéfico advindo do contato pele a pele, visto que a prática está intrinsicamente associada a promoção da amamentação na primeira hora pós parto, visando o estabelecimento e manutenção do AME, assim como nenhum dos outros benefícios fisiológicos tanto para os recém nascidos como para as puérperas foram apontados (SAMPAIO ARR, et al., 2016; SILVA CM, 2016).

Tendo em vista que o contato pele a pele proporciona para a mãe benefícios como diminuição da dor causada pelo ingurgitamento mamário, liberação de ocitocina que aumenta a eficácia na contração uterina no pós-parto imediato, assim previne hemorragias por atonia uterina e para o RN benefícios como, melhora da efetividade da primeira mamada e redução do tempo para haver sucção efetiva, melhora da estabilidade cardiorrespiratória, melhora no índice de aleitamento materno nos primeiros quatro meses de vida com maior duração da amamentação, além da melhora termodinâmica citada, evidenciou-se uma lacuna do conhecimento sobre os benefícios fisiológicos para mãe e bebê, devido a restrição das falas voltadas apenas para recuperação térmica do neonato (SAMPAIO ARR, et al., 2016). 


\section{Conforto e vínculo representado pelo contato pele a pele}

O conforto e vínculo vivenciado pelo $\mathrm{RN}$ e a parturiente, acompanhado pela equipe de enfermagem nas salas de parto, da maternidade em questão, como benefício do contato pele a pele foi relatado nas respostas obtidas na pesquisa.

"Estreitar os laços do mesmo (recém-nascido) mediante o contato pele a pele" (E5)

"Para o bem-estar da mãe e do bebê" (E1)

"Proporcionar ao RN alento, conforto e aconchego" (E4)

"Acalma muito a insegurança da mãe e tranquiliza o RN." (E3)

$\mathrm{O}$ aspecto psíquico do momento da chegada de um filho vivenciado pela mãe é individual e único. $O$ profissional da saúde tem papel ativo e essencial no primeiro contato entre RN e a mãe, que chega para parir com uma história, expectativas, medos, ansiedade e posteriormente a chegada do bebê (SAMPAIO ARR, et al, 2016). O contato pele a pele imediato tem como benefício para ambas as partes - mãe e bebê, o aumento do vínculo entre os dois (BRASIL, 2013).

Após um intenso trabalho de parto, mediante as dores das contrações e neste contexto institucional as preocupações advindas do estado geral de saúde da parturiente por ter passa por uma gestação de alto risco e assim ter patologias associadas ao parto, o contato pele a pele se apresenta como uma recompensa positiva dessa trajetória (SILVA BAA e BRAGA LP, 2019).

De acordo com estudo realizado em maternidade pública no interior da Bahia, o quarto passo da IHAC tem se tornado um ato mecânico para cumprimento de protocolo porque a lógica da assistência é baseada na produção de procedimentos, diminuindo a qualidade desse momento primordial para o binômio e tão esperado pela genitora (SANTOS M, 2014).

Se os profissionais visualizam um benefício psíquico em ambos, eles preconizam a realização do procedimento independente do fluxo do hospital. O conhecimento, mesmo que simplificado nas respostas obtidas, permite que seja avaliada a importância que o profissional dá para o procedimento em questão, por ser algo que está relacionado com o emocional tanto de quem está vivenciando a situação, como de quem está auxiliando e fazendo a assistência do binômio, porém, vale ressaltar que a pesquisa não aponta qual a autonomia do profissional da enfermagem mediante a tomada de decisões na sala de parto.

\section{O conhecimento e a prática da técnica do contato pele a pele em $\mathrm{RN}$ de alto risco}

O conhecimento e a aplicação da técnica do contato pele a pele, são explorados nessa seção, mostrando que os profissionais que participaram da pesquisa conhecem como se faz, mas não tem bem definido quando fazê-lo.

"É o primeiro contato da mãe com o bebê, contato da pele do RN em contato com a pele da mãe" (E1)

"Contato pele a pele é a experiência que o RN tem nas primeiras horas" (E5)

"Colocar o RN após o nascimento em contato com a mãe" (E5)

"Quando possível, seria muito bom para ambos." (E3)

"Depende das condições do parto e do RN" (E1)

Identifica-se colocação vaga de quando aplicar a técnica. Julgando-se pelas outras categorias já abordadas, o conhecimento do profissional da enfermagem, que caracteriza o perfil dos funcionários da maternidade da instituição em questão, não tem sido atualizado para melhor fixação da fundamentação da aplicação do contato pele a pele. Entende-se que o contato pele a pele é o contato da pele nua da mãe com a pele nua do RN, sem barreiras, mas para melhor adesão da técnica em partos de alto risco, é visivelmente necessário maior conhecimento sobre o assunto, para valorização de seus efeitos positivos (ALBUQUERQUE CC, et al., 2008)

"Conhecimento que tenho adquirido mediante palestras [...] e reforço por parte da chefia [...] da importância do mesmo" (E4) 
Esta fala nos permite detectar a importância da educação permanente e a educação continuada em saúde. Sendo a educação permanente condição de desenvolvimento pessoal potencializado, a fim da promoção da capacidade técnica específica, a aquisição de novos conhecimentos, conceitos e atitudes - é o aprender constante. A educação continuada é um conjunto de experiências subsequentes à formação inicial adquirida através de metodologias formais para a atualização do conhecimento (PASCHOAL AS, et al., 2007)

$O$ fato de a participante relatar que o hospital fornece palestras sobre o assunto e a chefia de enfermagem "reforçar" a importância da técnica demonstra que os gestores estão preocupados e empenhados para que seus funcionários tenham seu conhecimento sempre renovado e atualizado, porém, sem avaliação do sucesso das intervenções educacionais, o que é primordial para a transformação profissional e consequentemente a qualidade do atendimento ao usuário prestado pelo mesmo. Um fator limitador do estudo foi à participação das funcionárias, com objetivo de maior adesão o questionário foi adaptado para um formulário, mesmo assim a adesão dos profissionais para com a pesquisa foi baixa, o que foi inesperado tratando-se de um hospital universitário.

\section{CONCLUSÃO}

Na pesquisa observaram-se lacunas em relação ao conhecimento e prática do contato pele a pele no pósparto imediato, mesmo assim, a técnica é efetuada corretamente quando avaliada a sua possibilidade de acontecer. Infere-se a necessidade de investimentos na educação continuada na área da saúde materno infantil, sobre o contato pele a pele, ressaltando a importância dessa pratica para benefícios imediatos e futuros para parturiente e neonato, e assim ter uma melhor aplicação da teoria na prática cotidiana dos profissionais de forma fundamentada. Espera-se que este estudo contribua para uma reflexão aprofundada por parte do profissional da área de enfermagem, sobre o contato pele a pele em relação a sua prática e benefícios, mesmo em partos classificados como de alto risco.

\section{REFERÊNCIAS}

1. ALBUQUERQUE CC, et al. Sistematização da Assistência de Enfermagem a um Binômio Mãe-lactentes Utilizando a Teoria das Necessidades Humanas Básicas e a Cipe. Cienc. Cuid. Saude, 2008; 7(3):392-398.

2. BARDIN L. Análise de conteúdo. 3rd ed. Lisboa. Edições 70; 2011.

3. BRASIL. Ministério da Saúde. Além da Sobrevivência: Práticas integradas de atenção ao parto, benéficas para a nutrição e a saúde de mães e crianças. 2011; 1a edição: 50.

4. BRASIL. Ministério da Saúde. Atenção à saúde do recém-nascido - guia para profissionais de saúde - cuidados gerais. 2012; $2^{2}$ edição:35.

5. BRASIL. Ministério da Saúde. Iniciativa Hospital Amigo da criança. 2014.

6. BRASIL. Ministério da Saúde. Além da sobrevivência: práticas integradas de atenção ao parto, benéficas para a nutrição e a saúde de mães e crianças. 2013. Primeira edição. 16-18 p.

7. COCA KP, et al. Conjunto de medidas para o incentivo do aleitamento materno exclusivo intra-hospitalar: evidências de revisões sistemáticas. 2018; 36(2). 214:220.

8. Sociedade Brasileira de Pediatria. 2016. Reanimação do recém-nascido maior de 34 semanas em sala de parto.

9. LAMOUNIER JA. Promoting and supporting breast-feeding: Baby Friendly Hospital Initiative. Jornal de Pediatria. 1996; 363:368.

10. LAMOUNIER JÁ, et al. Iniciativa hospital amigo da criança: 25 anos de experiência no Brasil. Rev. paul.2019; 37(4).

11. MATOS TA, et al. Contato precoce pele a pele entre mãe e filho: significado para mães e contribuições para a enfermagem. Rev. Bras. Enferm. 2010; 63(6):998:1004.

12. PASCHOAL AS, et al. Percepção da educação permanente, continuada e em serviço para enfermeiros de um hospital de ensino. Rev. esc. enferm. USP. 2007; 41(3): 478:484.

13. ROSA HR, et al. Mães alojadas: alojamento conjuntos no hospital geral como forma de humanização. Bol. - Acad. Paul. Psicol. 2016; 36 (90): 141:156.

14. SAMPAIO ARR, et al. Contato pele a pele ao nascer: um desafio para a promoção do aleitamento materno em maternidade pública no Nordeste brasileiro com o título de Hospital Amigo da Criança. Epidemiol. Serv. Saúde. 2016; 25(2): $281: 290$.

15. SANTOS M, et al. Vivenciando o contato pele a pele com o recém-nascido no pós-parto como um ato mecânico. Revista Brasileira de Enfermagem. 2014; 67 (2):7:202.

16. SILVA BAA, BRAGA LP. Fatores promotores do vínculo mãe-bebê no puerpério imediato hospitalar: uma revisão integrativa. Rev. Sociedade Brasileira de Psicologia Hospitalar. 2019; 22 (1): 258:279.

17. SILVA CM, et al. Fatores associados ao contato pele a pele entre mãe/filho e amamentação na sala de parto. Rev. Nutr. 2016; 29 (4): 457:471.

18. SOARES T, et al Prevalência da hipotermia na primeira hora de vida de prematuros com peso $\leq 1500 \mathrm{~g}$. Rev Gaúcha Enferm. 2020;41(esp).

19. WHO (World Health Organization). National. Implementation of the Baby-friendly Hospital Initiative. Geneva, 2017.

20. WHO (Organização Mundial da Saúde). The ten steps to Successful Breastfeeding. Genebra, 2018. 